\title{
A note on utility-based pricing
}

\author{
Mark H.A. Davis*and Daisuke Yoshikawa ${ }^{\dagger}$
}

August 4, 2010

\begin{abstract}
In incomplete market theory, the utility-based price and the indifference pricing have especially received much attention in pricing methods using utility function. This paper constructs the framework to unite these two methods and analyzes the relationship between them, using the setting of the exponential utility. Furthermore, we deduce the equilibrium price under the framework of the utility-based price.
\end{abstract}

\section{Introduction}

When pricing a random endowment in the incomplete market, the framework based on the utility maximization principle has recently received much attention and intensively developed. Particularly, utility indifference price and utility-based price are two major frameworks. Both of them are based on utility optimization problem; however, the setting is different from each other. Utility indifference framework is given as the problem, where the price of the random endowment is given by the threshold price for the expected utility to be constant for a given quantity of the random endowment for selling (or, buying). On the other hand, the utility-based price is given by the problem, where, for a fixed price, each investor maximizes his expected utility by optimizing the quantity of the random endowment. That is, this quantity is the solution of the optimization problem. The utility-based price is given as the price consistent with this optimized quantity. In this paper, we make the relation between these two frameworks clear. This trial gives us a new insight to the framework based on the utility maximization; that is, we find that these frameworks can be used for not only pricing the random endowment, but also capturing an effect of the change of the price. Furthermore, similar to Davis and Yoshikawa(2010)[2] where the equilibrium price is deduced under the utility indifference framework, the equilibrium price is deduced in the framework of the utility-based price.

This paper breaks down into 2 parts. In the first part, we set up the model and set the tool for utility indifference framework and utility-based price, using the exponential utility function. This tool enables us to analyze the effect of the change of the price of the random endowment. In the second part, we consider the relation between the utility indifference price and the utility-based price and deduce the equilibrium under the framework of the utility-based price.

\section{The Model}

The mathematical framework is given by the filtered probability space $(\Omega, \mathcal{F}, \mathbb{F}, P)$, where $\mathbb{F}:=\left(\mathcal{F}_{t}\right)_{0 \leq t \leq T}$, $\mathcal{F}:=\mathcal{F}_{T}$, and $\mathcal{F}_{0}$ is trivial. Stochastic process $X \in \mathbb{R}^{d}$ is defined as semimartingale, and the expected value of $X$ is given by the probability measure $P$. Consider the $\mathcal{F}_{T}$-measurable random variable $B$ which will generate some payoff at time $T$. The random variable $B$ is assumed unbounded from below (Delbaen et al.(2002)[3]) and we assume that $\mathbb{E}\left[e^{(\alpha+\epsilon) B}\right]<\infty$ and $\mathbb{E}\left[e^{-\epsilon B}\right]<\infty$ for some fixed $\alpha, \epsilon \in(0, \infty)$ (Becherer(2003)[1]).

Utility indifference price is defined as the price of the random endowment $B$ which equates the maximized expected utility of a terminal wealth without the random endowment $B$ and the maximized

\footnotetext{
*Imperial College London

†Imperial College London
} 
expected utility of a terminal wealth with the random endowment $B$. That is, if the price $p$ is the utility indifference price, then it satisfies,

$$
\sup _{\theta \in \Theta} \mathbb{E}\left[U\left(x+\int_{0}^{T} \theta_{t}^{\top} d X_{t}\right)\right]=\sup _{\theta \in \Theta} \mathbb{E}\left[U\left(x-p q+\int_{0}^{T} \theta_{t}^{\top} d X_{t}+B q\right)\right]
$$

where $q \in \mathbb{R}$ (hereafter, we call this quantity as 'utility indifference quantity'). Let $\theta:=\left\{\theta_{t} ; t \in[0, T]\right\} \in$ $\Theta$ be $\mathbb{R}^{d}$-valued admissible trading strategy and $\Theta$ be the set of $X$-integrable and predictable processes. The left hand side of the above equation is a maximized expected utility not including the random endowment $B$ and the right hand side is a maximized expected utility including $B$. When $q>0$, the price $p$ is called the utility indifference sell price. Otherwise, it is called the utility indifference buy price. Hereafter, we specify the utility function $U(\cdot)$ as

$$
U(x)=-e^{-\gamma x}
$$

where $\gamma \in \mathbb{R}_{+}$is risk-aversion.

We construct a technique to graphically specify the relation between utility indifference prices and utility indifference quantities. First, we define $u(\theta, q ; \gamma):=\ln \mathbb{E}\left[e^{-\gamma\left(\int_{0}^{T} \theta_{t}^{T} d X_{t}+B q\right)}\right]$. From this definition, it is easily deduced that $u_{q q} \geq 0$ which is second order differential on $q$. Using this function, the expected utility of the right hand side of (1) is written as follows,

$$
u(\theta, q ; \gamma)-\gamma(x-p q)=\ln \left(-\mathbb{E}\left[U\left(x-p q+\int_{0}^{T} \theta_{t}^{\top} d X_{t}+B q\right)\right]\right) .
$$

The utility indifference framework for $q \in \mathbb{R}$ is rewritten,

$$
\begin{aligned}
\inf _{\theta \in \Theta} u(\theta, 0 ; \gamma)-\gamma x & =\inf _{\theta \in \Theta}\{u(\theta, q ; \gamma)-\gamma(x-p q)\} \\
& =\inf _{\theta \in \Theta}\{u(\theta, q ; \gamma)\}-\gamma(x-p q) .
\end{aligned}
$$

This shows that the problem of an expected utility maximization is independent of the initial capital (in the left hand side, the initial capital is $x$, and in the right hand side, it is $x-p q)$. Let $\theta^{q}$ be the solution of $\inf _{\theta \in \Theta}\{u(\theta, q ; \gamma)\}$. Note that the solution $\theta^{q}$ is unique by the convexity of the expected utility. From (2), the utility indifference price $p^{U I}(B ; q)$ is given such as,

$$
p^{U I}(B ; q)=\frac{1}{\gamma q}\left(u\left(\theta^{0}, 0 ; \gamma\right)-u\left(\theta^{q}, q ; \gamma\right)\right) .
$$

That is,

$$
u\left(\theta^{q}, q ; \gamma\right)=u\left(\theta^{0}, 0 ; \gamma\right)-\gamma p^{U I}(B ; q) q
$$

Furthermore, we define the function $u(q ; \gamma)$ as follows,

$$
u(q ; \gamma):=\inf _{\theta \in \Theta}\{u(\theta, q ; \gamma)\}=u\left(\theta^{q}, q ; \gamma\right) .
$$

We call this function as an indifference curve. Using the indifference curve, (3) is rewritten as,

$$
u(q ; \gamma)=u(0, \gamma)-\gamma p^{U I}(B ; q) q .
$$

The right hand side of the above equation is a linear function of $q$. We can specify a shape of the left hand side of this equation by next three lemmas.

\section{Lemma 2.1}

An indifference curve $u(q ; \gamma)$ for $q \in \mathbb{R}$ is the envelope curve of $u\left(\theta^{q}, \tilde{q} ; \gamma\right)$, where $\tilde{q} \in \mathbb{R}$. That is, the indifference curve is contact with $u\left(\theta^{q}, \tilde{q} ; \gamma\right)$ at $\tilde{q}=q$ 
Proof From the definition of $\theta^{q}, u\left(\theta^{q}, q ; \gamma\right) \leq u(\theta, q ; \gamma)$ for all $\theta \in \Theta$. For all $\epsilon>0, u\left(\theta^{q}, q+\epsilon ; \gamma\right) \geq$ $u\left(\theta^{q+\epsilon}, q+\epsilon ; \gamma\right)=u(q+\epsilon ; \gamma)$ and $u\left(\theta^{q}, q-\epsilon ; \gamma\right) \geq u\left(\theta^{q-\epsilon}, q-\epsilon ; \gamma\right)=u(q-\epsilon ; \gamma)$. Equality in the former case holds if and only if $\theta^{q}=\theta^{q+\epsilon}$. In the latter case, equality holds if and only if $\theta^{q}=\theta^{q-\epsilon}$. It shows that the indifference curve $u(q ; \gamma)$ contacts with the $u\left(\theta^{q}, t ; \gamma\right)$ at $t=q$.

Q.E.D.

\section{Lemma 2.2}

The indifference curve is convex function.

Proof For $q, q^{\prime} \in \mathbb{R}$ and $0 \leq k \leq 1$,

$$
\begin{aligned}
k u(q ; \gamma)+(1-k) u\left(q^{\prime} ; \gamma\right) & =k u\left(\theta^{q}, q ; \gamma\right)+(1-k) u\left(\theta^{q^{\prime}}, q^{\prime} ; \gamma\right) \\
& =k \ln \mathbb{E}\left[e^{-\gamma\left(\int_{0}^{T}\left(\theta_{t}^{q}\right)^{\top} d X_{t}+B q\right)}\right]+(1-k) \mathbb{E}\left[e^{-\gamma\left(\int_{0}^{T}\left(\theta_{t}^{q^{\prime}}\right)^{\top} d X_{t}+B q^{\prime}\right)}\right] \\
& =\ln \mathbb{E}\left[e^{-\gamma\left(\int_{0}^{T}\left(\theta_{t}^{q}\right)^{\top} d X_{t}+B q\right)}\right]^{k} \mathbb{E}\left[e^{-\gamma\left(\int_{0}^{T}\left(\theta_{t}^{q^{\prime}}\right)^{\top} d X_{t}+B q^{\prime}\right)}\right]^{1-k} \\
& \geq \ln \mathbb{E}\left[e^{-k \gamma\left(\int_{0}^{T}\left(\theta_{t}^{q}\right)^{\top} d X_{t}+B q\right)} e^{-(1-k) \gamma\left(\int_{0}^{T}\left(\theta_{t}^{q^{\prime}}\right)^{\top} d X_{t}+B q^{\prime}\right)}\right] \\
& =\ln \mathbb{E}\left[e^{-\gamma\left(\int_{0}^{T}\left(k \theta_{t}^{q}+(1-k) \theta_{t}^{q^{\prime}}\right)^{\top} d X_{t}+B\left(k q+(1-k) q^{\prime}\right)\right)}\right] \\
& =u\left(k \theta^{q}+(1-k) \theta^{q^{\prime}}, k q+(1-k) q^{\prime} ; \gamma\right) \\
& \geq u\left(\theta^{k q+(1-k) q^{\prime}}, k q+(1-k) q^{\prime} ; \gamma\right)=u\left(k q+(1-k) q^{\prime} ; \gamma\right) .
\end{aligned}
$$

We use Hölder's inequality on line 4.

Q.E.D.

We define $\mathcal{M}$ as a set of $\Theta$-martingale measures satisfying $H(Q \mid P)<\infty$. And $H(Q \mid P):=\int \frac{d Q}{d P} \ln \frac{d Q}{d P} d P$ is relative entropy of $Q$ with respect to $P$, whcih is always non negative (c.f. Theorem 1.4.1 of Ihara(1993)[5]). Hereafter, we assume that

$$
\mathcal{M} \neq \emptyset \text {. }
$$

We write the solution of $\inf _{Q \in \mathcal{M}} H[Q \mid P]$ as $Q^{0} \in \mathcal{M}$ which we call minimal entropy martingale measure (hereafter, MEMM).

\section{Lemma 2.3}

The slope of a tangent line to an indifference curve $u(q ; \gamma)$ at $q=0$ is given by $-\gamma \mathbb{E}^{Q^{0}}[B]$.

Proof From Theorem 3.2, Lemma 3.3 of Davis and Yoshikawa(2010)[2] and the definition of $u(\theta, q ; \gamma)$,

$$
-\left.\frac{1}{\gamma} \frac{\partial u\left(\theta^{0}, q ; \gamma\right)}{\partial q}\right|_{q=0}=\mathbb{E}\left[B \frac{e^{-\gamma\left(\int_{0}^{T}\left(\theta_{t}^{0}\right)^{\top} d X_{t}\right)}}{\mathbb{E}\left[e^{-\gamma\left(\int_{0}^{T}\left(\theta_{t}^{0}\right)^{\top} d X_{t}\right)}\right]}\right]=\mathbb{E}^{Q^{0}}[B] .
$$

Q.E.D.

From these Lemmas, the shape of the indifference curve is specified. Figure 1 shows it. For simplicity, let $p^{*}=\mathbb{E}^{Q^{0}}[B]$. The bold line in Figure 1 is indifference curve. The utility indifference quantity $q^{1}$ corresponding to the utility indifference price $p^{1}$ is given by the intersection between the line $u(0 ; \gamma)-\gamma p^{1} q$ and $u(q ; \gamma)$. Likewise, the utility indifference quantity $q^{2}$ to $p^{2}$ is given by the intersection between $u(0 ; \gamma)-\gamma p^{2} q$ and $u(q ; \gamma)$. Once the quantity of $B$ is given, through the indifference curve, we can find the corresponding indifference price. Conversely, if some price is given, then we can find the corresponding utility indifference quantity which does not change the expected utility, i.e., when the price changes from $p^{1}$ to $p^{2}$, if the investor changes the strategy from $q^{1}$ to $q^{2}$ on the indifference curve, the expected utility is invariant. 


\section{The relationship between indifference pricing and utility-based price}

The utility indifference framework requires that the quantity of the random endowment is given. Under this constraint, the investor offers the rational price according to the utility maximization principle. This price is utility indifference price. However, we can consider the situation in which the investor optimizes the quantity of the random endowment under the constraint that the price is given. Under this situation, the definition of the utility-based price is given. According to Hugonnier and Kramkov(2004)[4], " $a$ utility-based price for $f$ (it is a $N$-dimensional random endowment with some payoff at $t=T$ ) is defined as a vector $\tilde{p}(x, q) \in \mathbb{R}^{N}$ such that the agent's holdings $q$ in the claims are optimal in the model where the claims can be traded at time 0 at price $\tilde{p}(x, q)$." In this section, we consider the utility-based price in the case of exponential utility. From the above definition, it is clear that the utility-based price is given as the solution of the utility maximization problem about the quantity $q$; that is, for some given price $p$, we have to consider the problem,

$$
\inf _{\theta \in \Theta, q \in \mathbb{R}}\{u(\theta, q ; \gamma)-\gamma(x-p q)\} .
$$

It is a natural expansion of the right hand side of (2). This problem is solved as follows,

$$
\begin{aligned}
\inf _{\theta \in \Theta, q \in \mathbb{R}}\{u(\theta, q ; \gamma)-\gamma(x-p q)\} & =\inf _{q \in \mathbb{R}}\left\{\inf _{\theta \in \Theta}\{u(\theta, q ; \gamma)\}+\gamma p q\right\}-\gamma x \\
& =\inf _{q \in \mathbb{R}}\left\{u\left(\theta^{q}, q ; \gamma\right)+\gamma p q\right\}-\gamma x \\
& =\inf _{q \in \mathbb{R}}\{u(q ; \gamma)+\gamma p q\}-\gamma x
\end{aligned}
$$

For the quantity $q$ to be optimal, it has to satisfy

$$
\frac{\partial u(q ; \gamma)}{\partial q}=-\gamma p
$$

That is, the optimal $q$ is given by the quantity at which a slope of the tangent line to the indifference curve $u(q ; \gamma)$ is given by $-\gamma p$. For given $p$, let $q^{p}$ be satisfying (7). Conversely, for a given quantity $q$, if the price $p$ satisfies $(7)$, it is a utility-based price. And, we write such a price as $p^{H K}(B ; q)$.

\section{Remark 3.1}

Figure 2 shows this situation. Let $p^{0}$ be an initial market price of $B$. We assume that the investor optimizes his expected utility; that is, he chooses the strategy to hold the quantity $q^{0}$ where the slope of the tangent line to the indifference curve is $-\gamma p^{0}$. In this sense, the price $p^{0}$ is the utility-based price $p^{H K}\left(B ; q^{0}\right)$.

\section{Proposition 3.2}

For a given price $p$, if the investor's strategy is optimal in the sense satisfying (7), the expected utility is larger than the expected utility under the strategy of utility indifference framework.

Proof For a given price $p$, using $q^{p}$ satisfying (7),

$$
\begin{aligned}
\inf _{\theta \in \Theta, q \in \mathbb{R}}\{u(\theta, q ; \gamma)-\gamma(x-p q)\} & =u\left(q^{p} ; \gamma\right)+\gamma p q^{p}-\gamma x \\
& =u(0 ; \gamma)-\gamma p^{U I}\left(B ; q^{p}\right) q^{p}+\gamma p q^{p}-\gamma x \\
& \leq u(0 ; \gamma)-\gamma x=\inf _{\theta \in \Theta} u(\theta, 0 ; \gamma)-\gamma x .
\end{aligned}
$$

From the convexity of indifference curve, if $q^{p}<0,0>-p^{U I}\left(B ; q^{p}\right) \geq-p$. On the other hand, if $q^{p}>0,-p \geq-p^{U I}\left(B ; q^{p}\right)$. This is used to deduce the inequality of $(8)$. The right hand side of $(8)$ is corresponding to the expected utility without $B$. 
This proposition implies that introducing the random endowment $B$ in the market gives positive effect for every investor. The following theorem shows the relation between utility indifference price and the utility-based price more clearly.

\section{Theorem 3.3}

For some $q<0$, the utility-based price $p^{H K}(B ; q)$ is larger than the utility indifference price $p^{U I}(B ; q)$, and for $q>0$, the utility-based price $p^{H K}(B ; q)$ is less than the utility indifference price $p^{U I}(B ; q)$.

Proof It is clear from the convexity of the indifference curve.

Q.E.D.

From Theorem 3.3, $p^{H K}(B ; q)=p^{U I}(B ; q)$ for all $q \in \mathbb{R}$ if and only if the indifference curve is linear. However, for any indifference curve $u(q ; \gamma)$, the slope of the tangent line at $q=0$ is $-\gamma \mathbb{E}^{Q_{0}}[B]$. Therefore, in the case that an indifference curve is linear, the slope of this indifference curve has to be $-\gamma \mathbb{E}^{Q_{0}}[B]$. It means that the utility indifference price is given by $\mathbb{E}^{Q_{0}}[B]$ for all $q$. Since Proposition 3.2 of Becherer(2003)[1] shows that $\lim _{\gamma \downarrow 0} p^{U I}(B ; q)=\mathbb{E}^{Q^{0}}[B]$, the risk-aversion of the investor whose indifference curve is linear is zero.

We consider the generalization of the the utility indifference framework. That is, we generalize the initial state of the utility indifference pricing from not holding the random endowment to holding the random endowment with some initial price $p$ (generalized version of the utility indifference framework).

\section{Problem 3.4}

Let $p^{0}$ be the initial price. Assume that the investor takes a policy holding a quantity $q$ of the random endowment. Consider the price $p^{U I}(B ; q)$ satisfying as follows,

$$
\sup _{\theta \in \Theta, \tilde{q} \in \mathbb{R}} \mathbb{E}\left[U\left(x-p^{0} \tilde{q}+\int_{0}^{T} \theta_{t}^{\top} d X_{t}+B \tilde{q}\right)\right]=\sup _{\theta \in \Theta} \mathbb{E}\left[U\left(x-p^{U I}(B ; q) q+\int_{0}^{T} \theta_{t}^{\top} d X_{t}+B q\right)\right] .
$$

The left hand side of this problem includes the principle of utility-based price and the equality of this problem implies the utility indifference framework. The solution is given as follows,

$$
p^{U I}(B ; q)=\frac{1}{\gamma q}\left(u\left(q^{p^{0}} ; \gamma\right)-u(q ; \gamma)\right)+p \frac{q^{p^{0}}}{q}
$$

When $q^{p^{0}}=0$, this solution is consistent with the usual setting of the indifference pricing.

Using Figure 2, we consider this generalized version of the utility indifference framework. If the investor wants to change his policy from $q^{0}$ to $\tilde{q}^{1}, p^{1}$ is the price that the investor should offer for letting his expected utility be constant. It means that $p^{1}$ is the utility indifference price corresponding to the utility indifference quantity $\tilde{q}^{1}$; in fact, if the market price changes from $p^{0}$ to $p^{1}$, the investor can make his expected utility constant by choosing $\tilde{q}^{1}$ as the strategy ${ }^{1}$. Next, if the investor optimizes his expected utility for the price $p^{1}$, he will choose the strategy $q^{1}$ where the line with slope $-\gamma p^{1}$ contacts with the indifference curve. The result of the convexity of indifference curve is $\left|q^{1}\right| \leq\left|\tilde{q}^{1}\right|$. In the context of economics, the change of the demand (or supply) for the change of the price is divided into two parts; that is, the total effect of the price change is divided into a substitution effect and an income effect. As we have seen, the change from $q^{0}$ to $\tilde{q}^{1}$ is brought by Hicks substitution effect, because the expected utility is constant by the change from $p^{0}$ to $p^{1}$. The change from $\tilde{q}^{1}$ to $q^{1}$ is considered as the income effect. In fact, the change of the price makes the initial asset change from $x-p^{0} q^{0}$ to $x-p^{1} q^{1}$ (we consider the

\footnotetext{
${ }^{1}$ Consider the line with slope $-\gamma p^{0}$ through point $\left(q^{0}, u\left(q^{0} ; \gamma\right)\right)$. The intersection with this line and the vertical axis is given by $u\left(q^{0} ; \gamma\right)+\gamma p^{0} q^{0}$. Note that the $\log$ of negative value of the expected utility to the strategy $\left(p^{0}, q^{0}\right)$ is given by $u\left(q^{0} ; \gamma\right)-\gamma\left(x-p^{0} q^{0}\right)($ see $(6))$.

Next, considering the line with slope $-\gamma p^{1}$ thorough point $\left(\tilde{q}^{1}, u\left(\tilde{q}^{1} ; \gamma\right)\right.$. The intersection with this line and the vertical axis is given by $\left.u\left(\tilde{q}^{1} ; \gamma\right)+\gamma p^{1} \tilde{q}^{1}\right)$. Note that the $\log$ of negative value of the expected utility to the strategy $\left(p^{1}, \tilde{q}^{1}\right)$ is given by $u\left(\tilde{q}^{1} ; \gamma\right)-\gamma\left(x-p^{1} \tilde{q}^{1}\right)$ (see $\left.(2)\right)$.

In Figure 2, the intersections with the vertical axis for these two lines coincide. It implies that, by regulating the strategy from $q^{0}$ to $\tilde{q}^{1}$, the corresponding expected utility is constant through the change from $p^{0}$ to $p^{1}$.
} 


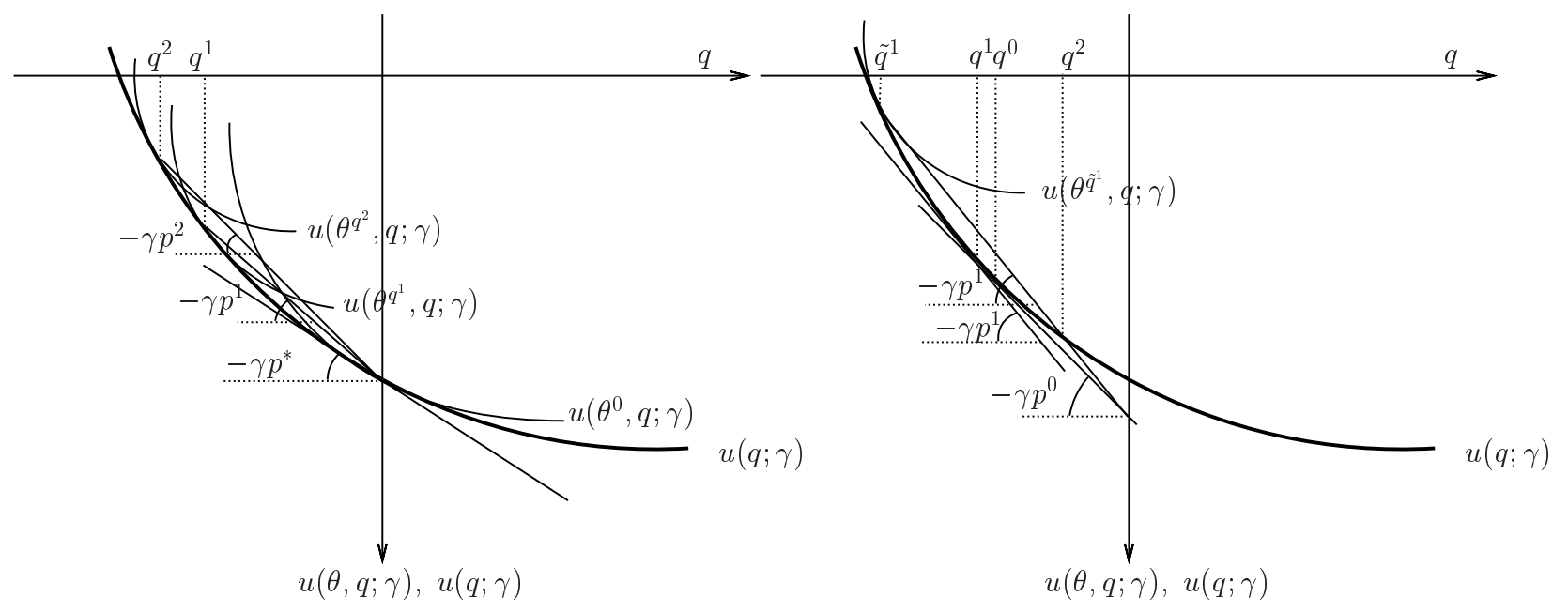

Figure 1: Utility indifference ptice, utility indiffer- Figure 2: Utility-based price and utility indifference ence quantity and indifference curve price based on Problem 3.4

"income" as an initial asset). This change is positive, since $\left(x-p^{1} q^{1}\right)-\left(x-p^{0} q^{0}\right) \geq 0$ by the convexity of the indifference curve. A goods is called superior goods, if the income effect is positive; 'positive' means that the demand increases when the income increases. Likewise, a goods is called inferior goods, if the income effect is negative. Therefore, if the investor chooses $\tilde{q}^{1}$ as the utility indifference quantity, the random endowment is superior goods. However, the investor can choose $q^{2}$ as the utility indifference quantity. In this case, the random endowment is inferior goods. That is, although the utility indifference price is unique, the utility indifference quantity is not necessarily unique when the initial state is not $\left(p^{0}, q^{0}\right)=\left(p^{*}, 0\right)$.

Davis and Yoshikawa(2010)[2] deduces the equilibrium under the utility indifference framework. Using the setting of the indifference curve, we can easily deduce the equilibrium even under the framework of the utility-based price. Following Davis and Yoshikawa(2010)[2], we give the definition of the equilibrium.

\section{Definition 3.5}

Let an economy specify the investors' preferences which is described by the utility function $U:=$ $\left\{U_{i}(\cdot) ; U_{i}(x):=-e^{-\gamma_{i} x}, i=1, \cdots, I+J\right\}$. An allocation $q^{s}:=\left\{q_{i}^{s}, i=1, \cdots, I\right\}, q^{b}:=\left\{q_{j}^{b}, j=1, \cdots, J\right\}$ and a price $p$ of the random endowment $B$ constitutes a price equilibrium if there is an assignment such that

1. Offer price condition: For any investor with utility function $\left\{U_{i}, i=1, \cdots, I\right\}$, when the investor sells $q_{i}^{s}$-units of the random endowment, $\left(p, q_{i}^{s}\right)$ is preferred to all other allocations $\left(p,\left(q_{i}^{s}\right)^{\prime}\right)$; that is, an expected utility corresponding to the allocation $\left(p, q_{i}^{s}\right)$ is larger than another expected utility corresponding to the allocation $\left(p,\left(q_{i}^{s}\right)^{\prime}\right)$.

2. Bid price condition: For any investor with utility function $\left\{U_{I+j}, j=1, \cdots, J\right\}$, when the investor buys $q_{j}^{b}$-units of the random endowment, $\left(p, q_{j}^{b}\right)$ is preferred to all other allocations $\left(p,\left(q_{j}^{b}\right)^{\prime}\right)$; that is, an expected utility corresponding to the allocation $\left(p, q_{j}^{b}\right)$ is larger than another expected utility corresponding to the allocation $\left(p,\left(q_{j}^{b}\right)^{\prime}\right)$.

3. Market cleared condition $\sum_{i=1}^{I} q_{i}^{s}=\sum_{j=1}^{J} q_{j}^{b}$.

From this definition, a theorem about equilibrium is deduced.

\section{Proposition 3.6}

If investors in the market of the random endowment $B$ act according to the utility maximization, then an equilibrium price is given by,

$$
p^{*}=\mathbb{E}^{Q^{0}}[B] .
$$

Furthermore, the equilibrium is zero trade equilibrium. 
Proof From (5) and (7), the optimal strategy of $q$ for the price $p^{*}=\mathbb{E}^{Q^{0}}[B]$ is 0 for every investor. Since the indifference curve is convex, the quantity $q$ optimal for $p>p^{*}$ is negative, vice versa. Since it is common for all investors, if $p>p^{*}$, selling (that is, $q<0$ ) is optimal for all investors, vice versa. Therefore, equilibrium is zero trade and equilibrium price is given by MEMM $Q^{0}$.

Q.E.D.

\section{References}

[1] D. Becherer. Rational hedging and Valuation of Integrated Risks under Constant Absolute Risk Aversion. Insuarance: Mathematics \& Economics, 33:1-28, 2003.

[2] M.A.H. Davis and D. Yoshikawa. An equilibrium approach to indifference pricing. working paper, 2010 .

[3] F. Delbaen, P. Grandits, Th. Rheinländer, D. Samperi, M. Schweizer, and Ch. Stricker. Exponential hedging and entropic penalties. Mathematical finance, 12(2):99-123, 2002.

[4] J. Hugonnier and D. Kramkov. Optimal investment with random endowments in incomplete markets. The Annals of Applied Probability, 14(2):845-864, 2004.

[5] S. Ihara. Information theory for continuous system. World Scientific, 1993. 